\title{
Design and Investigation into the Thermal and Mechanical Performance of a Polymer Composite Prototype Gas-Liquid Heat Exchanger
}

\author{
Bidisha Ghosh, Wadeema Yousef, Mariam Al Jaberi, Nuha Al Hajeri, Asrar Al Braiki, \\ Valerie Eveloy*, and Peter Rodgers \\ Department of Mechanical Engineering, the Petroleum Institute, Abu Dhabi, United Arab Emirates
}

\begin{abstract}
Seawater-cooled metallic heat exchangers used in natural gas processing are prone to corrosion and fouling, resulting in increased operational and maintenance costs. A lab-scale polymer composite gas-liquid webbed tube bank heat exchanger is designed and evaluated for application in conditions representative of a fielded natural gas processing application. The heat exchanger thermal performance and structural integrity are investigated numerically using computational fluid dynamics (CFD) and finite element (FE) models, respectively. For polymer composite thermal conductivities above 20 $\mathrm{W} / \mathrm{m}-\mathrm{K}$, in forced gas-side convection, the exchanger heat transfer rate is comparable to that of a high conductivity conventional metallic heat exchanger having the same geometry, at reduced materials, manufacturing and operational costs. In addition, the prototype heat exchanger would be structurally reliable at the maximum envisaged gas-side operating pressure for the application considered.
\end{abstract}

Keywords: Polymer, Composite, Heat Exchanger

\section{Introduction}

Heat exchangers are used in a variety of chemical and hydrocarbon processing applications, with seawater typically used as coolant in coastal Arabian Gulf industrial facilities. As a result of exposure to seawater and other corrosive fluids, currently used conventional metallic heat exchangers are prone to corrosion, erosion and fouling [1,2]. This can result in significant degradation of process cooling/heating capacity and reliability over time, and increased operational costs due to increased fluid pumping power, intensive maintenance requirements and/or costly corrosion/fouling mitigation approaches. With advancement in process technologies, the demand for improved gas-liquid heat exchangers made of innovative materials, that can mitigate the above operational challenges, has grown over the past decade. Recent studies [1,2] suggest that polymer composite materials enhanced with high thermal-conductivity fillers have excellent potential to replace metallic materials in gas-liquid or gas-gas heat exchanger applications, where the gas-side thermal resistance generally

${ }^{*}$ Corresponding author

E-mail: veveloy@pi.ac.ae

(c) 2016 International Association for Sharing Knowledge and Sustainability

DOI: $10.5383 /$ ijtee. 11.01 .008 exceeds that of the solid domain. In such applications, polymer heat exchangers could provide (i) improved resistance to corrosion and fouling, (ii) greater geometric design flexibility, hence exchanger performance, (iii) reduced materials, manufacturing and operational costs, as well as (iv) reduced weight relative to metallic exchangers.

In this study, a laboratory-scale, high-thermal conductivity polymeric heat exchanger is designed and prototyped for application in conditions representative of low-temperature, low-pressure natural gas refining processes. The heat exchanger design concept is derived from a review of commerciallyavailable heat exchangers made of standard (i.e., non-thermally enhanced) polymers and published heat exchanger studies. A numerical investigation of heat exchanger thermofluid performance and structural behavior is undertaken using computational fluid dynamics (CFD) and finite element (FE) analyses, respectively. Before presenting this analysis, an overview of both commercially-available polymer heat exchangers and published research for applications other than hydrocarbon processing is presented. 


\section{Background}

A compilation of commercially-available polymeric heat exchangers and their vendors is presented in Table 1. These products are categorized in terms of their geometry, material, applications, operating conditions and thermo-fluid performance. Industrial applications include heating, ventilation and air conditioning (HVAC), thermal ice storage, semiconductor processing, bio processes, and water treatment, to name a few. However, none of the vendors listed in Table 1 report hydrocarbon or petrochemical processing applications. In addition, for applications other than hydrocarbon processing, the polymer materials used appear to be in almost all instances, standard (i.e., non-thermally-enhanced) polymers with low thermal conductivity. Thus the most common polymer heat exchanger materials are non-thermally enhanced polypropylene (PP), polyvinylidene fluoride (PVDF), and polyethylene (PE). Hybrid polymer-metallic designs can also be found. The majority of heat exchanger designs are shell and tube and plate geometries from millimeter to meter scale, with heat exchanger overall dimensions varying significantly between vendors. When reported, heat exchanger $\mathrm{U}$-values range from approximately $46 \mathrm{~W} / \mathrm{m}^{2} \mathrm{~K}$ to $1500 \mathrm{~W} / \mathrm{m}^{2} \mathrm{~K}$, and pressure drops from 0.1 mbar to 3 bar. Operating temperatures are confined to approximately $-40^{\circ} \mathrm{C}$ to $+150^{\circ} \mathrm{C}$. Manufacturers' development efforts appear to focus on improved heat transfer efficiency, low-weight and compactness.

An overview of published studies on polymer heat exchangers is presented in Table 2 . The most commonly reported materials are standard (i.e., non-thermally enhanced) poly-ether-etherketone (PEEK) films and PE, for applications including seawater desalination, HVAC, and fuel cells. For plate film heat exchangers, the minimum polymer film thickness is $25 \mathrm{~m}$. Uvalues of up to $3800 \mathrm{~W} / \mathrm{m}^{2} \mathrm{~K}$ are reported with pressure drops of up to $4 \mathrm{kPa}$.

\section{Lab-scale Heat Exchanger Design and Prototyping}

Several design concepts were initially derived from commercially-available polymer heat exchanger products (Table 1) and published research for applications other than hydrocarbon processing (Table 2), as well as relevant standards [25]. The conceptual designs initially included tube and plate, spiral, concentric tube and finned tube bank geometries. The main design performance criteria to be evaluated include heat exchanger overall heat transfer coefficient (i.e., U-value) or heat transfer rate, gas- and liquid-side pressure drops, and structural and chemical compatibility with low-temperature (i.e., $\leq$ $120^{\circ} \mathrm{C}$ ), low-pressure (i.e., $\leq 8$ bar) operating conditions in natural gas processing, cost and life cycle environmental emissions. The maximum allowable operating temperature should be sufficient below the material heat deflection temperature (HDT).

The thermally-enhanced material selection was based on material vendor thermal conductivity, vendor mechanical properties (e.g., yield strength, tensile strength, impact strength) and injection moldability. Thermally enhanced polymers have thermal conductivities ranging from 1 to $30 \mathrm{~W} / \mathrm{m}-\mathrm{K}$ [2]. Based on preliminary analytical modeling, thermally enhanced material selection focused on thermal conductivities above 10 $\mathrm{W} / \mathrm{m}-\mathrm{K}$. Rodgers et al. [26] presented a review of the thermophysical properties of twenty seven commercially-available, thermally enhanced polymer composites from six leading vendors, from which two promising candidate groups of materials, namely polyphenylene sulfide (PPS) and polyimide 66 (PA66), were identified. PA 66 was found to have slightly better thermal conductivity, tensile strength, and flexural strength than PPS at room temperature. However, PA66 exhibited lower tensile modulus than PPS, and could be prone to hygroscopic swelling. Overall, PPS was considered the more promising polymeric composite material for heat exchanger applications. Based on the above considerations, thermally enhanced PPS and PA66 polymer composites were considered for further performance investigation in this study. Their material properties reported by three different commercial vendors are listed in Table 3 . The lab-scale prototype design part dimensions were constrained to $90 \%$ of the laboratory injection molding equipment shot size, $98 \mathrm{~cm}^{3}$, as per equipment vendor recommendations. The maximum operating gas and liquid approach velocities were determined to be $8 \mathrm{~m} / \mathrm{s}$ and 1 $\mathrm{m} / \mathrm{s}$, respectively, for typical application requirements.

Based on analytical predictions of heat transfer rate and pressure drop for the above conceptual design geometries considered, a webbed (i.e., finned) tube and plate design was selected, the geometry of which is depicted in Figure 1. Unlike in unfinned tube banks, the liquid channels are joined by planar wall segments (i.e., fins) for enhanced heat transfer, resulting in both the gas and liquid being unmixed. The fins are placed on the gas-side, as it contributes most of the total gas-liquid thermal resistance chain.

\section{Numerical Analysis}

The lab-scale heat exchanger prototype (Figure 1) thermofluid performance and structural integrity were numerically assessed using computational fluid dynamics (CFD) and finite element (FE) analyses, respectively. The ANSYS Workbench platform software was used for model construction and analysis. Geometry construction and meshing were undertaken using the DesignModeler [27] and Meshing [28] modules, respectively, with the CFD and FE models solved using the Fluent [29] and Mechanical [30] modules, respectively.

\section{Thermofluid performance}

The lab-scale heat exchanger CFD model is illustrated in Figure 2. The heat exchanger air- and water-side thermofluid processes and conductive domain were modeled at approach air flow velocities of 2 to $8 \mathrm{~m} / \mathrm{s}$ and a water flow velocity of $1 \mathrm{~m} / \mathrm{s}$. The lengths of the air- and water manifolds/piping extended $0.1 \mathrm{~m}$ upstream of the heat exchanger test section (with fully developed flow conditions prescribed at the piping inlets) and $0.25 \mathrm{~m}$ downstream of the air- and water-side test section outlets. A uniform inlet air temperature of approximately $60^{\circ} \mathrm{C}$ and water inlet temperature of $20^{\circ} \mathrm{C}$ were imposed. The air and water fluid domains were modeled using the standard high Reynolds k- $\varepsilon$ turbulent model in conjunction with the software enhanced wall treatment. This near-wall modeling method combines a two-layer zonal model with enhanced wall functions for improved accuracy over standard law-of-the-wall wall functions. The computational mesh is a non-conformal hybrid triangular/tetrahedral mesh having approximately 3 million elements, Figure 2(b). Solution mesh independence was assessed and mesh adaptation was applied to refine the near-wall mesh to impose a near-wall cell non-dimensional coordinate $(\mathrm{y}+)$ of approximately 1 . The default software convergence criteria were applied. 
Ghosh et al. / Int. J. of Thermal \& Environmental Engineering, 11 (2016) 51-59

Table 1: Commercially-Available Polymer Heat Exchangers

\begin{tabular}{|c|c|c|c|c|c|c|}
\hline Company & Geometry & Material & Application & Dimensions & $\begin{array}{l}\text { Operating } \\
\text { conditions }\end{array}$ & $\begin{array}{l}\text { Thermofluid } \\
\text { performance }\end{array}$ \\
\hline $\begin{array}{c}\text { AB Segerfröjd } \\
{[3]}\end{array}$ & Plate & $\begin{array}{l}\text { Extruded PP } \\
\text { sheets }\end{array}$ & $\begin{array}{l}\text { Ventilation, } \\
\text { humidification, } \\
\text { electronics cooling, } \\
\text { wet flue gas } \\
\text { recuperation } \\
\end{array}$ & $\mathrm{t}=2 \mathrm{~mm}-9 \mathrm{~mm}$ & $\mathrm{~T}=-40^{\circ} \mathrm{C}$ to $90^{\circ} \mathrm{C}$ & $\begin{array}{c}\mathrm{U}=46 \mathrm{~W} / \mathrm{m}^{2} \mathrm{~K} \\
\Delta \mathrm{P}=0.0001- \\
0.004 \text { bar }\end{array}$ \\
\hline $\begin{array}{l}\text { AIL Research } \\
{[4]}\end{array}$ & Plate & $\begin{array}{c}\text { N/R }(\mathrm{k} \sim 0.3 \\
\mathrm{W} / \mathrm{m}-\mathrm{K})\end{array}$ & $\begin{array}{c}\text { Corrosive liquid } \\
\text { cooling }\end{array}$ & $\begin{array}{c}\mathrm{t}=0.127 \mathrm{~mm} \\
\text { Wall thickness }=0 \\
76 \mathrm{~m}\end{array}$ & $\begin{array}{c}\mathrm{T} \leq 148^{\circ} \mathrm{C} \\
\mathrm{P} \leq 2.75 \text { bar }\end{array}$ & N/R \\
\hline AMETEK [5] & $\begin{array}{l}\text { Shell and } \\
\text { tube }\end{array}$ & $\begin{array}{l}\text { FEP, PFA, } \\
\text { PVDF, High } \\
\text { purity (HP), or } \\
\text { Q-Series }\end{array}$ & $\begin{array}{l}\text { Temperature control } \\
\text { of laboratory baths, } \\
\text { small production } \\
\text { tanks }\end{array}$ & $\begin{array}{c}\text { Compact frame } \\
\text { sizes }=0.305 \times 0.305 \\
\mathrm{~m} \text { to } 0.457 \times 0.457 \mathrm{~m} \\
\mathrm{~A}=0.195 \mathrm{~m}^{2} \text { to } \\
0.929 \mathrm{~m}^{2}\end{array}$ & $\begin{array}{c}\mathrm{T}=6^{0} \mathrm{C} \text { to } 295^{\circ} \mathrm{C} \\
\mathrm{P} \leq 11 \mathrm{bar}\end{array}$ & N/R \\
\hline $\begin{array}{c}\text { CALORPLAS } \\
\text { T } \\
\text { Warmetechnik } \\
{[6]} \\
\end{array}$ & $\begin{array}{l}\text { Shell and } \\
\text { tube, tube } \\
\text { plate }\end{array}$ & $\begin{array}{l}\text { PVDF, PP, PE- } \\
\text { RT, PFA }\end{array}$ & $\begin{array}{l}\text { Heating/cooling of } \\
\text { highly corrosive } \\
\text { media }\end{array}$ & $\begin{array}{c}\mathrm{W}=0.305 \mathrm{~m} \\
\mathrm{~L}=0.518-2.53 \mathrm{~m} \\
\text { Tubes } \mathrm{OD}=6 \mathrm{~mm} \\
\& \text { ID } 4.8=\mathrm{mm}\end{array}$ & $\begin{array}{c}\mathrm{T}=-30^{\circ} \mathrm{C} \text { to } \\
+135^{\circ} \mathrm{C} \\
\mathrm{P} \leq 16 \text { bar }\end{array}$ & $\begin{array}{c}\mathrm{U}=221-238 \\
\mathrm{~W} / \mathrm{m}^{2} \mathrm{~K} \\
\Delta \mathrm{P}=0.05-0.5 \\
\text { bar }\end{array}$ \\
\hline $\begin{array}{c}\text { Fluorotherm } \\
\text { [7] }\end{array}$ & $\begin{array}{l}\text { Shell and } \\
\text { tube }\end{array}$ & $\begin{array}{c}\text { Shell is PVC } \\
\text { and CPVC } \\
\text { Tubing is PTFF, } \\
\text { FEP and PFA }\end{array}$ & $\begin{array}{l}\text { Semiconductor, } \\
\text { biotechnology, } \\
\text { environmental, } \\
\text { product finishing }\end{array}$ & $\mathrm{A}=0.093-9.3 \mathrm{~m}^{2}$ & $\begin{array}{c}\text { Shell and tube: } \\
\mathrm{T}=60^{\circ} \mathrm{C} \text { to } 93^{\circ} \mathrm{C} \\
\mathrm{P}=2.76 \text { bar } @ 60 \\
{ }^{\circ} \mathrm{C}\end{array}$ & N/A \\
\hline Greenbox [8] & Plate & PVC & N/R & $\begin{array}{c}\text { Wall thickness < } \\
0.5 \mathrm{~mm}\end{array}$ & $\begin{array}{c}\mathrm{T}=-20^{\circ} \mathrm{C} \text { to } 60^{\circ} \mathrm{C} \\
\mathrm{P} \leq 0.01 \text { bar }\end{array}$ & $\begin{array}{c}\mathrm{U}=24.3 \\
\mathrm{~W} / \mathrm{m}^{2} \mathrm{~K} \\
\Delta \mathrm{P}=0.005 \text { bar }\end{array}$ \\
\hline HeatMatrix [9] & N/R & $\begin{array}{c}\text { Stacked or } \\
\text { integrated } \\
\text { hybrid (metal } \\
\text { and polymer) }\end{array}$ & $\begin{array}{l}\text { Applications } \\
\text { susceptible to cold } \\
\text { spot corrosion }\end{array}$ & N/R & N/R & N/R \\
\hline $\begin{array}{l}\text { Magen Eco- } \\
\text { Energy [10] }\end{array}$ & $\begin{array}{l}\text { Shell and } \\
\text { tube, tube- } \\
\text { plate }\end{array}$ & $\begin{array}{c}\text { PP, PVDF, PE- } \\
\text { RT }\end{array}$ & $\begin{array}{c}\text { Corrosive materials } \\
\text { processing in biogas, } \\
\text { chemical and metal } \\
\text { industries }\end{array}$ & $\mathrm{N} / \mathrm{R}$ & $\begin{array}{c}\text { Flow rate }=0.139 \\
\mathrm{~m}^{3} / \mathrm{s}\end{array}$ & N/R \\
\hline Polytetra [11] & $\begin{array}{l}\text { Shell and } \\
\text { tube, } \\
\text { Suspended } \\
\text { plug-in, } \\
\text { Circular, } \\
\text { hybrid, } \\
\text { compact } \\
\end{array}$ & $\begin{array}{l}\text { PFA, ECTFE, } \\
\text { PTFE, PVDF } \\
\text { flouroplastics }\end{array}$ & $\begin{array}{c}\text { Galvanization, } \\
\text { chemical, wastewater, } \\
\text { foodstuffs and textile } \\
\text { processes }\end{array}$ & N/R & N/R & N/R \\
\hline $\begin{array}{c}\text { Process } \\
\text { Technology } \\
{[12]}\end{array}$ & $\begin{array}{l}\text { Shell and } \\
\text { tube, Coil }\end{array}$ & $\begin{array}{l}\text { Shell: PP of } \\
\text { PVDF } \\
\text { Tube: PFA }\end{array}$ & $\begin{array}{l}\text { Immersion } \\
\text { applications }\end{array}$ & $\begin{array}{c}\text { Wall thickness }= \\
0.76 \mathrm{~mm}\end{array}$ & $\begin{array}{c}\text { Shell: } \mathrm{P} \leq 2.06 \text { bar } \\
\text { at } 82^{\circ} \mathrm{C} \\
\text { Tube: } \mathrm{P} \leq 2.41 \text { bar } \\
\text { at } 120^{\circ} \mathrm{C}\end{array}$ & $\begin{array}{c}\mathrm{U}=142-284 \\
\mathrm{~W} / \mathrm{m}^{2} \mathrm{~K}\end{array}$ \\
\hline PolyCoil [13] & $\begin{array}{l}\text { Shell and } \\
\text { tube, flat } \\
\text { panel array }\end{array}$ & $\begin{array}{l}\text { Nylon - } \\
\text { Polyamide }\end{array}$ & $\begin{array}{l}\text { Military vehicles, } \\
\text { small pleasure crafts, } \\
\text { HVAC, thermal ice } \\
\text { storage, } \\
\text { evaporative coolers }\end{array}$ & $\begin{array}{c}\mathrm{t}=0.02 \mathrm{~m} \\
\text { Total dimensions }= \\
1.973 \times 0.279 \times 0.076 \\
\mathrm{~m} \\
\text { Tubing dimensions }= \\
1.829 \times 0.279 \times 0.076 \\
\mathrm{~m}\end{array}$ & $\begin{array}{c}\mathrm{T}=-40{ }^{0} \mathrm{C} \text { to }+149 \\
{ }^{0} \mathrm{C} \\
\mathrm{P} \leq 5.17 \text { bar } \\
\mathrm{V}_{\text {air }} \leq 2-10 \mathrm{~m} / \mathrm{s}\end{array}$ & $\begin{array}{c}\mathrm{U}=75 \mathrm{~W} / \mathrm{m}^{2} \mathrm{~K} \\
\Delta \mathrm{P}=0.07-0.4 \\
\text { bar }\end{array}$ \\
\hline TMW [14] & $\mathrm{N} / \mathrm{R}$ & $\mathrm{N} / \mathrm{R}$ & Water treatment & $\mathrm{N} / \mathrm{R}$ & $\mathrm{N} / \mathrm{R}$ & $\mathrm{N} / \mathrm{R}$ \\
\hline Note: & $\begin{array}{l}\text { at transfer surf } \\
\text { ner diameter ( } \\
\text { rmal conducti } \\
\text { gth }(\mathrm{m}), \\
\text { Jot reported, } \\
\text { uter diameter } \\
\text { sure (bar), } \\
\text { eat exchanger } \\
\text { nperature }\left({ }^{\circ} \mathrm{C}\right) \\
\text { t thickness }(\mathrm{m} \\
\text { erall heat trans } \\
\text { ir velocity }(\mathrm{m}\end{array}$ & $\begin{array}{l}\text { e area }\left(\mathrm{m}^{2}\right) \\
\mathrm{y}(\mathrm{W} / \mathrm{m}-\mathrm{K}), \\
\text { essure drop (bar), } \\
\text {, coefficient (W/m }\end{array}$ & & \multicolumn{3}{|c|}{$\begin{array}{l}\text { W = Width }(\mathrm{m}), \\
\text { CPVC = Chlorinated polyvinyl chloride, } \\
\text { FEP = ECTFE = Ethylene chlor-tri-fluoro-ethylene, } \\
\text { Fluorinated ethylene propylene, } \\
\text { PE = Polyethylene, } \\
\text { PE-RT = Polyethylene of raised temperature, } \\
\text { PFA = Perfluoroalkoxy alkane, } \\
\text { PP = Polypropylene, } \\
\text { PTFF = Polytetrafluoroethylene, } \\
\text { PVC = Polyvinyl chloride, } \\
\text { PVDF = Polyvinylidene fluoride }\end{array}$} \\
\hline
\end{tabular}


Ghosh et al. / Int. J. of Thermal \& Environmental Engineering, 11 (2016) 51-59

Table 2: Published Studies on Polymer Heat Exchangers

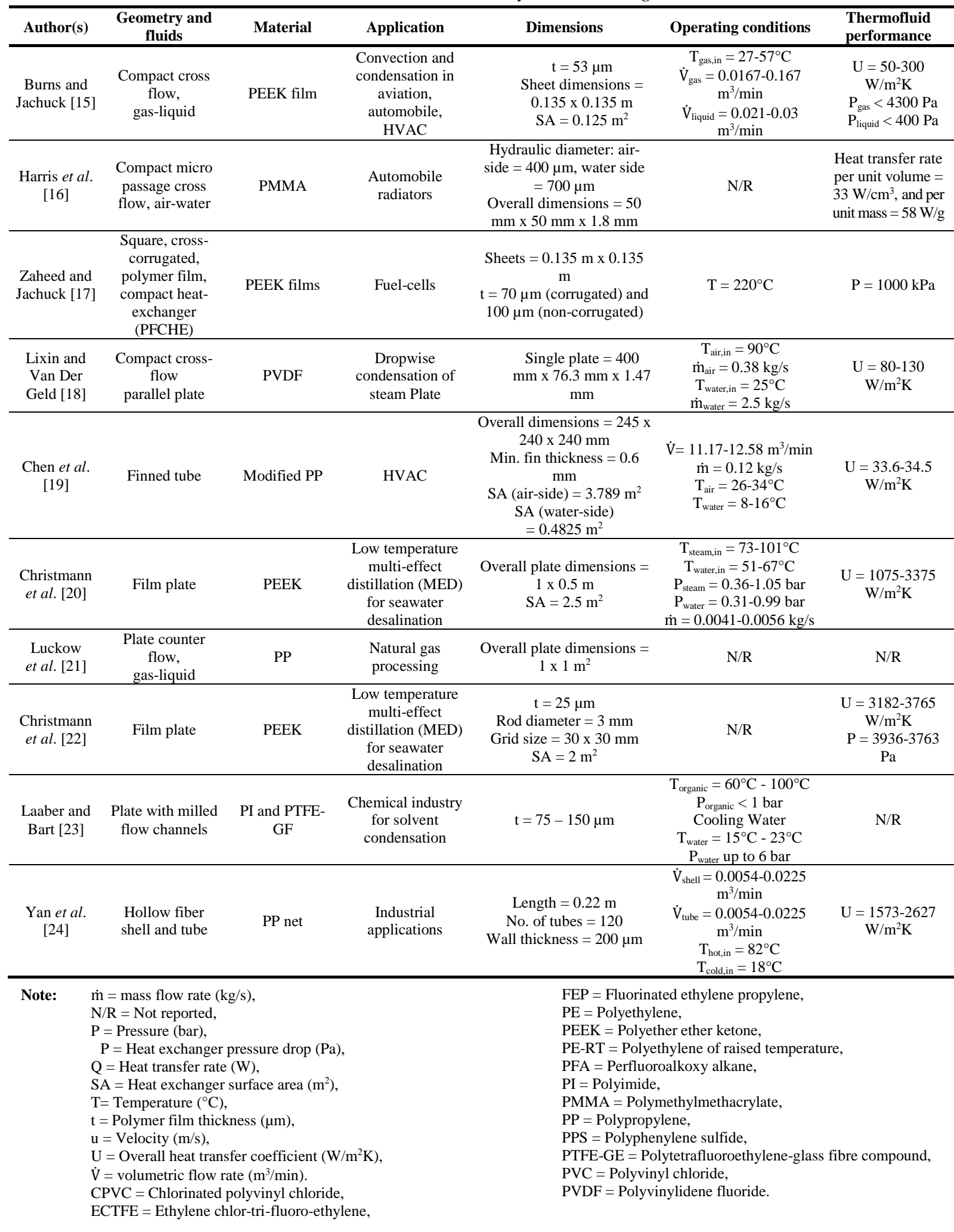


Table 3: Commercially Available Thermally Enhanced Polyphenylene Sulfide (PPS) and Polyamide 66 (PA66) Composite Materials Properties and their Injection Molding Characteristics

\begin{tabular}{|c|c|c|c|c|c|c|}
\hline \multirow[b]{2}{*}{ Property } & \multicolumn{3}{|c|}{ PA66 } & \multicolumn{3}{|c|}{ PPS } \\
\hline & V1 & $\mathrm{V2}$ & V3 & V1 & $\mathbf{V 2}$ & $\mathbf{V 3}$ \\
\hline (notched section) & $3.0 \mathrm{~kJ} / \mathrm{m}^{2}$ & NR & $27 \mathrm{~J} / \mathrm{m}$ & 27 & $2.0 \mathrm{~kJ} / \mathrm{m}^{2}$ & $27 \mathrm{~J} / \mathrm{m}$ \\
\hline (unnotched section) & $7.0 \mathrm{~kJ} / \mathrm{m}^{2}$ & NR & $133 \mathrm{~J} / \mathrm{m}$ & NR & $4.0 \mathrm{~kJ} / \mathrm{m}^{2}$ & $80 \mathrm{~J} / \mathrm{m}$ \\
\hline Tensile Modulus (MPa) & 14800 & 10600 & 18961 & 27600 & 13000 & 24132 \\
\hline Tensile strength (MPa) & 65 & 50 & 117 & 55.2 & 45 & 62 \\
\hline Flexural modulus (MPa) & 13000 & 10000 & 17238 & 22100 & 13000 & 18967 \\
\hline Flexural strength (MPa) & 90 & 70 & 193 & 82.7 & 70 & 86 \\
\hline $\begin{array}{l}\text { Thermal conductivity (in plane) } \\
(\mathrm{W} / \mathrm{m}-\mathrm{K})\end{array}$ & 20 & 20 & 32 & 19 & 20 & 20 \\
\hline $\begin{array}{l}\text { Heat deflection temperature } \\
\left({ }^{\circ} \mathrm{C}\right) @ 1.8 \mathrm{MPa}\end{array}$ & 245 & NR & NR & NR & 248 & 260 \\
\hline Melt Temperature $\left({ }^{\circ} \mathrm{C}\right)$ & $275-300$ & $232-282$ & $272-299$ & $320-340$ & $310-330$ & $307-329$ \\
\hline Mold Temperature $\left({ }^{\circ} \mathrm{C}\right)$ & $80.0-105$ & $38-93$ & $66-107$ & $140-160$ & $135-180$ & $135-177$ \\
\hline Drying time (hr) & 4.0 & $4.0-8.0$ & 4.0 & $4.0-6.0$ & $4.0-6.0$ & 6.0 \\
\hline Drying temperature $\left({ }^{\circ} \mathrm{C}\right)$ & 80.0 & 80.0 & 79.0 & $140-150$ & 105 & 149 \\
\hline Injection pressure (MPa) & NR & $5.2-13.8$ & $69-124$ & NR & $6.2-13.8$ & $69-103$ \\
\hline
\end{tabular}

Note: V1, V2 and V3 refer to vendor designation. NR = Not reported.

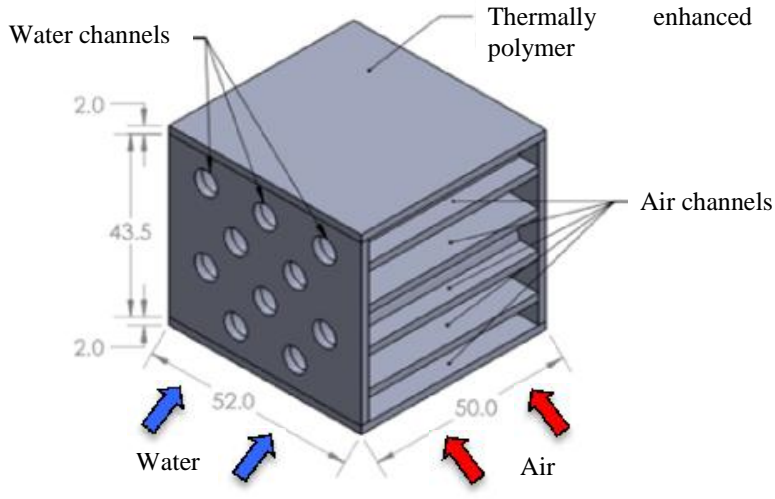

(a) Geometry

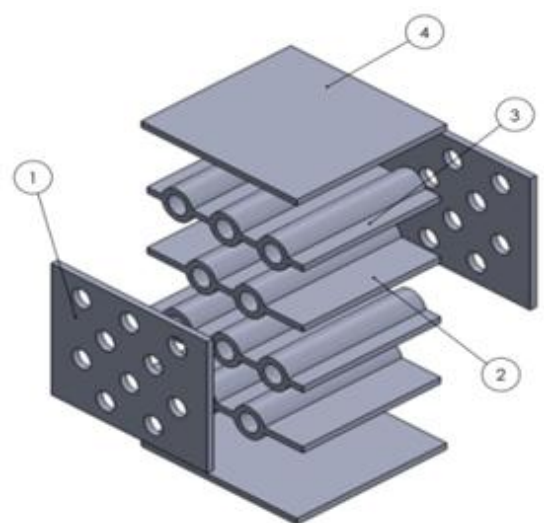

Note: $1=$ perforated wall, $2 \& 3=$ webbed tube rows, $4=$ upper wall (b) Exploded view

Note: Prototype overall external dimensions, $50 \mathrm{~mm}$ x $52 \mathrm{~mm}$ x 47.5mm. Solid wall thickness, $2 \mathrm{~mm}$. Liquid channel inner and outer diameters, 6.25 and $10.26 \mathrm{~mm}$, respectively. Liquid channel horizontal and vertical spacing, 16 and $8 \mathrm{~mm}$, respectively.

Figure 1: Lab-Scale Air-Water Prototype Heat Exchanger Geometry (all dimensions in mm)

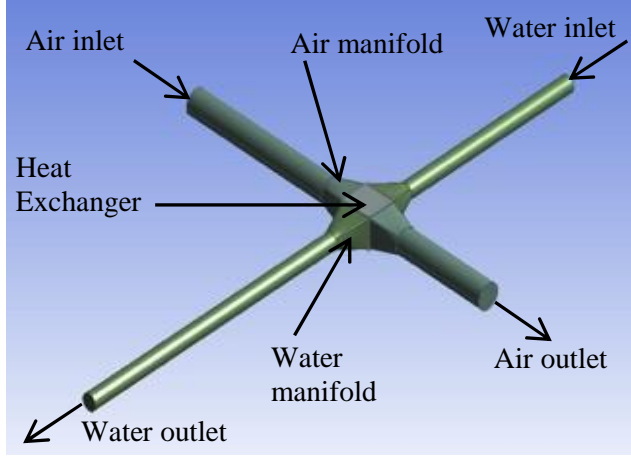

(a) Computational domain

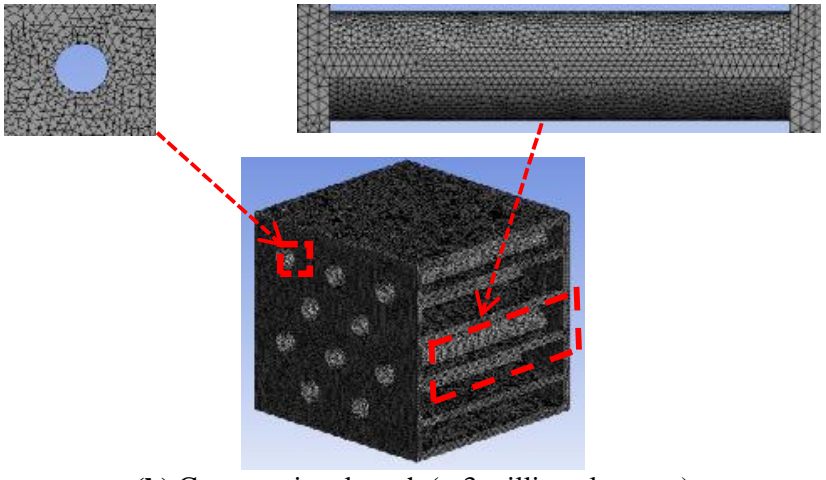

(b) Computational mesh ( 3 million elements)

Figure 2: CFD Model of Prototype Heat Exchanger 
The computed air- and water-side temperature fields for an airflow velocity of $4 \mathrm{~m} / \mathrm{s}$ are shown in Figure 3 . The average air temperature reduces by approximately $17^{\circ} \mathrm{C}$ from channel inlet to outlet, Figure 3(b), while the water temperature remains approximately constant, owing to the negligible water-side thermal resistance and large heat capacitance rate of water.

The predicted exchanger heat transfer rate is presented in Figure 4(a) as a function of forced airflow velocity, for several solid thermal conductivities spanning standard (i.e., nonthermally enhanced) polymers $(\mathrm{k}=0.3 \mathrm{~W} / \mathrm{m}-\mathrm{K})$, to thermallyenhanced polymer composites $(\mathrm{k}=5-30 \mathrm{~W} / \mathrm{m}-\mathrm{K})$, to highly conductive metallic alloys $(\mathrm{k}=100-200 \mathrm{~W} / \mathrm{m}-\mathrm{K})$. These predictions suggest that for thermal conductivities above approximately $20 \mathrm{~W} / \mathrm{m}-\mathrm{K}$, the exchanger heat transfer rate is similar to that of high conductivity metallic exchangers. It should be noted that metallic heat exchanger materials applied in natural gas processing corrosive environments have thermal conductivities typically less than $50 \mathrm{~W} / \mathrm{m}-\mathrm{K}$. Figure 4(b) presents the CFD predicted pressure drop as a function for forced airflow velocity.

\section{Structural Integrity}

The structural reliability of the design in Figure 1 was investigated based on the von Mises stress ( $\sigma \mathrm{v})$ distribution and associated deformation within the solid structure, to assist in identifying potentially weak areas in the structure. The FE model geometry and mesh are illustrated in Figure 5. A uniform pressure boundary condition of 8 bar was applied on all internal air-side channel internal surfaces, while the waterside pressure was set at $1 \mathrm{~atm}$. The computational mesh shown in Figure 5 was refined in regions of expected high pressure gradients, based on a solution mesh independence analysis.

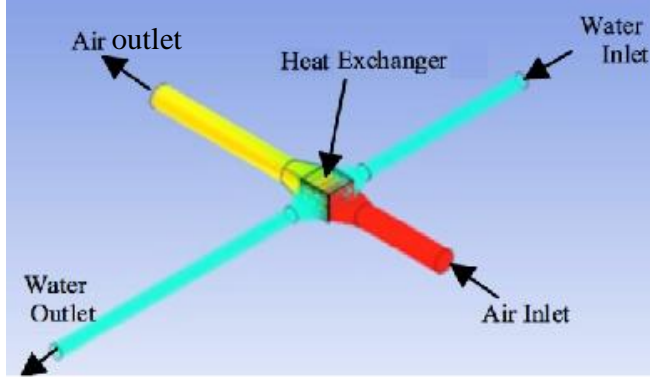

(a) Air- and water sides
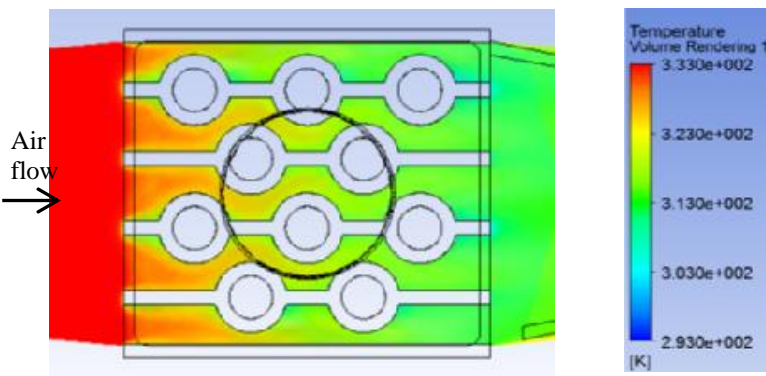

(b) Air channels

Note: PPS solid material thermal conductivity, $20 \mathrm{~W} / \mathrm{m}-\mathrm{K}$. Inlet airflow velocity and temperature of approximately of $4 \mathrm{~m} / \mathrm{s}$ and $60^{\circ} \mathrm{C}$, respectively. Water flow velocity and inlet temperature of $1 \mathrm{~m} / \mathrm{s}$ and $20^{\circ} \mathrm{C}$, respectively.

Figure 3: CFD Predicted Temperature Distributions in Heat Exchanger Air- and Water Channels

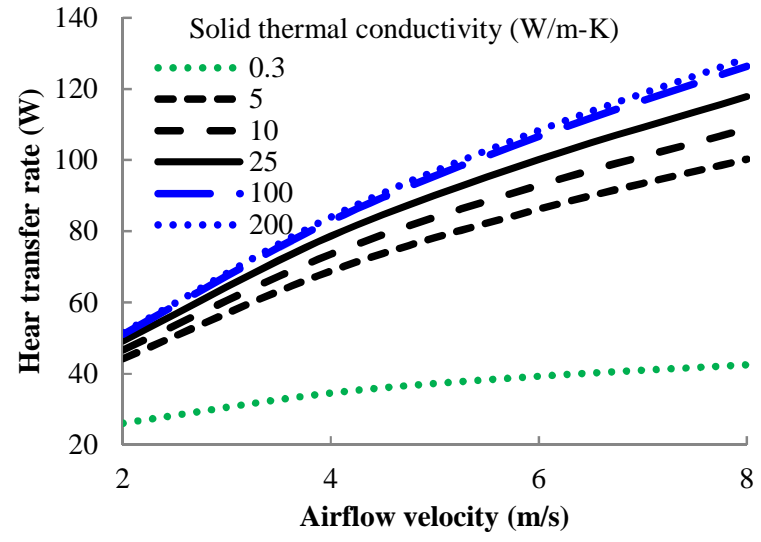

(a) Heat transfer rate as a function of solid thermal conductivity ranging from a standard (i.e., non-thermally enhanced) polymer $(k=$ $0.3 \mathrm{~W} / \mathrm{m}-\mathrm{K})$, to thermally-enhanced polymer composites $(k=5-30$ $\mathrm{W} / \mathrm{m}-\mathrm{K})$, to metallic alloys $(\mathrm{k}=100-200 \mathrm{~W} / \mathrm{m}-\mathrm{K})$

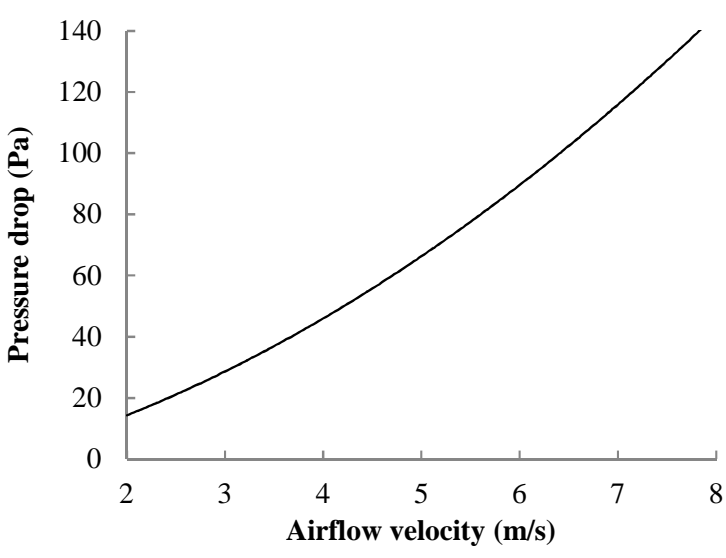

(b) Air-side pressure drop

Figure 4: CFD Predicted Heat exchanger Thermal and Hydraulic Performance 
Table 4: Thermally Enhanced Polyphenylene Sulfide (PPS) and Polyamide 66 (PA66), and Aluminum Material Properties for Structural modeling

\begin{tabular}{ccccc}
\hline Material Property & Density $\left(\mathbf{k g} / \mathbf{m}^{\mathbf{3}}\right)$ & Poisson's Ratio & Young's Modulus (GPa) & Tensile Yield Strength (MPa) \\
\hline PA66 & 1560 & 0.35 & 10.6 & 50 \\
PPS & 1700 & 0.35 & 13 & 45 \\
Aluminum & 2770 & 0.33 & 71 & 280 \\
\hline
\end{tabular}

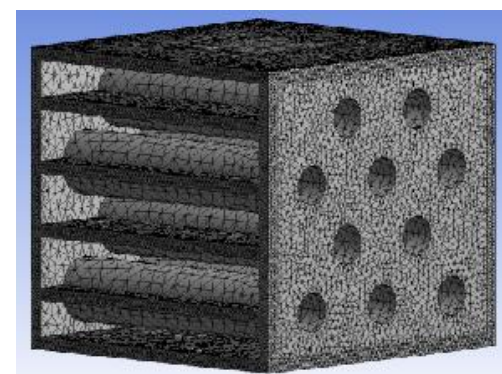

Figure 5: Heat Exchanger FE Model geometry and Computational Mesh (102,024 elements)
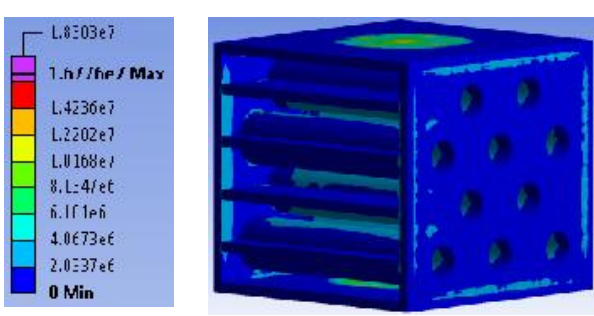

(a) Von Mises Stress, PPS
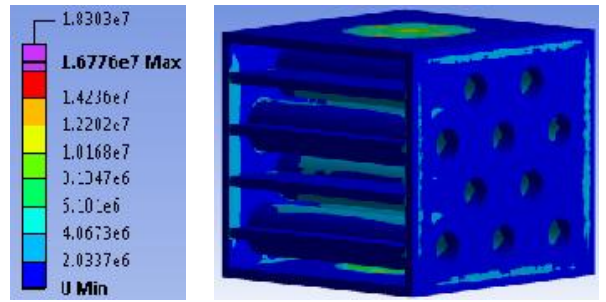

(c) Von Mises Stress, PA66
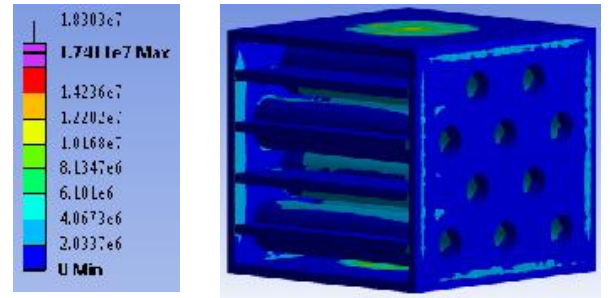

(e) Von Mises Stress, Aluminum
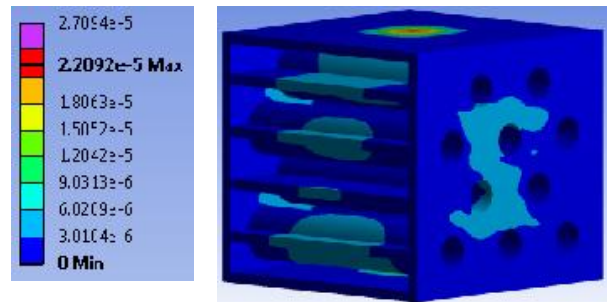

(b) Total Deformation, PPS
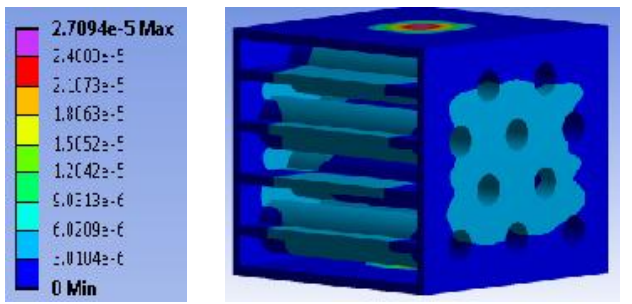

(d) Total Deformation, PA66
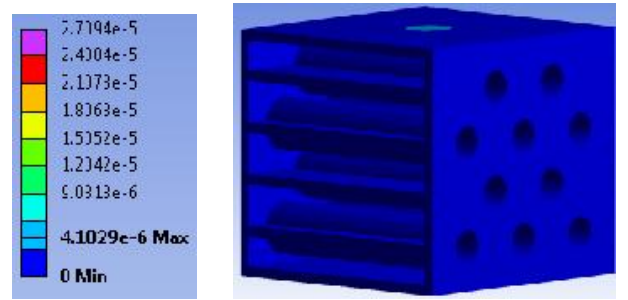

(f) Total Deformation, Aluminum's

Note: Uniform pressure of 8 bar applied to inner surface of all air channels.

Figure 6: FE Predictions of Von Mises Stress $\left(\sigma_{v}\right)$ and Total Deformation Distributions in Heat Exchanger

The model was solved for three different thermally enhanced polymer materials, namely PA66, PPS and aluminum. The properties of PPS and PA66 listed in Table 4 were prescribed. The predicted $\sigma_{\mathrm{v}}$ and total deformation distributions are shown in Figure 6. For all the materials under analysis, the location of the maximum $\sigma \mathrm{v}$ and total deformation is found to be at the center of the upper and lower heat exchanger surfaces, which are not supported by the heat exchanger manifold. The water tubes are not found to experience significant deformation. The maximum $\sigma_{\mathrm{V}}$ values are similar $(\sim 16.8 \mathrm{MPa})$ for PPS and PA66, which is attributable to their close Young's modulus and Poisson's ratio values (Table 4), whereas the aluminum structure has a slightly higher $\sigma \mathrm{v}(\sim 17.4 \mathrm{MPa})$. The maximum total deformation is lower for aluminum, approximately $4 \times 10^{-}$ 
${ }^{6} \mathrm{~m}$, than for the polymers, namely $2.2 \times 10^{-5} \mathrm{~m}$ for PPS and $2.7 \times 10^{-5} \mathrm{~m}$ for PA66. The predicted maximum $\sigma_{\mathrm{V}}$ values, which are less than the thermally enhanced polymer material yield strengths in Table 4 , and the predicted negligible maximum total deformation $\left(\sim 2.7 \times 10^{-5} \mathrm{~m}\right)$, indicate that the design would be structurally reliable. At higher gas-side pressures, the center of the upper and bottom exchanger surfaces may require to be supported to reduce the stress concentration in these areas.

\section{Conclusions}

Standard (i.e., non-thermally enhanced) commerciallyavailable polymer heat exchangers are currently applied to gasliquid or gas-gas applications other than hydrocarbon or petrochemical processing. In this study the design, prototyping and thermal/mechanical performances of a lab-scale gas-liquid heat exchanger made of a thermally enhanced polymer composite was explored in conditions representative of fielded low-temperature, low-pressure natural gas processing applications. Computational fluid dynamics (CFD) modeling indicates that a webbed tube bank design made of thermally enhanced polyphenylene sulfide (PPS) (thermal conductivity $20 \mathrm{~W} / \mathrm{m}-\mathrm{K}$ ) would offer a heat transfer rate close to that of its aluminum counterpart. In addition, finite element analysis (FEA) suggests that at high gas-side pressures, the heat exchanger structure would experience minimal mechanical deformation. Future work will further evaluate performance experimentally for a broader range of polymer materials and operational conditions. Such a design could significantly reduce materials and manufacturing costs compared to metallic exchangers, as well as pumping and maintenance costs, hence life cycle energy consumption and emissions.

\section{Acknowledgments}

The support of the Department of Mechanical Engineering, The Petroleum Institute, and Abu Dhabi National Oil Company (ADNOC) R\&D Gas Sub-Committee are gratefully acknowledged.

\section{References}

[1] Bar-Cohen, A. Rodgers, P. and Cevallos, J.G. "Application of Thermally Enhanced Thermoplastics to Seawater-Cooled Liquid-Liquid Heat Exchangers," in Proceedings of the Fifth European Thermal-Sciences Conference, Eindhoven, Netherlands, Paper No. HEX-10, 2008.

[2] Cevallos, J.G. Bergles, A.E. Bar-Cohen, A. Rodgers P. and Gupta. S.K. "Polymer Heat Exchangers-History, Opportunities, and Challenges," Heat Transfer Engineering, Vol. 33, no. 13, pp. 1075-1093, May 2012.

[3] AB Segerfröjd, "MONOBLOCK - The Polymer Heat Exchanger," Available: http://www.segerfrojd.com [Accessed: September 15, 2015].

[4] AIL Research, Inc. "Plastic Interchange Heat Exchanger," Available: http://ailr.com/our-technology/plasticinterchange-heat-exchanger/ [Accessed: September 15, 2015].

[5] AMETEK Fluoropolymer Products, "Minicoils FEP, PFA, High Purity (HP), PVDF, and Q-Series Heat Exchangers," Available:
http://www.ametekfpp.com/Immersion-coil/FEP-andPFA-minicoils.aspx [Accessed: September 15, 2015].

[6] CALORPLAST Warmetechnik, "Plastic Heat Exchangers," Available: http://www.calorplastwaermetechnik.de/en/products/plastic-heat-exchangers/ [Accessed: September 15, 2015].

[7] Fluorotherm, "Shell and Tube Heat Exchangers," Available: http://www.fluorotherm.com/products/heatexchangers/shell-tube-heat-exchangers/ [Accessed: September 15, 2015].

[8] Greenbox, "Air to Air Plate Heat Exchangers," Available: www.greenbox.uk.com [Accessed: October 10, 2014].

[9] HeatMatrix, "Products," Available:

http://heatmatrixgroup.com/products/\#aph [Accessed: September 15, 2015].

[10] Magen Eco Energy, "Industrial heat exchangers," Available: http://www.magen-ecoenergy.com/Industrial-HeatExchangers.aspx?c0=13280\&bsp=13278 [Accessed: June 13, 2015].

[11] Polytetra, "Plastic Heat Exchangers," Available: http://www.polytetra.de/en/products/heatexchanger.html [Accessed: September 15, 2015].

[12] Process Technology, "Industrial Heat Exchangers, Heating and Cooling Coils and Accessories by Process Technology," Available:

http://www.processtechnology.com/industrial-heatexchangers.html [Accessed: September 15, 2015].

[13] Polycoil, "Polycoil Heat Exchangers," Available: http://www.polycoil.eu/downloads/PolyCoil\%20EN\%20 1.3.pdf [Accessed: September 15, 2015].

[14] TMW, "Ecostill - Plastic Heat Exchanger," Available: http://uk.tmw-technologies.com/plastic-heat-exchanger/ [Accessed: September 15, 2015]

[15] Burns, J.R. and Jachuck, R.J.J. "Condensation Studies using Cross-Corrugated Polymer Film Compact Heat Exchanger," Applied Thermal Engineering, Vol. 21, pp. 495-510, 2001.

[16] Harris, C. Despa, M. and Kelly K., "Design and Fabrication of a Cross Flow Micro Heat Exchanger," Journal of Microelectromechanical Systems, Vol. 9, Issue 4, pp. 502-508, December 2009.

[17] Zaheed, L. and Jachuck R.J.J., "Performance of a Square, Cross-Corrugated, Polymer Film, Compact, HeatExchanger with Potential Application in Fuel Cells," Journal of Power Sources, Vol. 140, Issue 2, pp. 304-310, February 2005.

[18] Lixin, C. and Van Der Geld, C. "Experimental Study of Heat Transfer and Pressure Drop Characteristics of Air/Water and Air-Steam/Water Heat Exchange in a Polymer Compact Heat Exchanger," Heat Transfer Engineering, Vol. 26, Issue 2, pp.18-27, March 2005.

[19] Chen, L. Li, Z. and Guo, Z.-Y. "Experimental Investigation of Plastic Finned-Tube Heat Exchangers, with Emphasis on Material Thermal Conductivity," Experimental Thermal and Fluid Science, Vol. 33, Issue 5, pp. 922-928, July 2009.

[20] Christmann, J.B. P. Krätz, L.J. Bart, H.-J. "Novel Polymer Film Heat Exchangers for Seawater 
Desalination," Desalination and Water Treatment, Vol. 21, Issue 1-3, pp. 162-174, September 2010.

[21] Luckow, P. Bar-Cohen, A. Rodgers, P. and Cevallos J., "Energy Efficient Polymers for Gas-Liquid Heat Exchangers," Transactions of the ASME, Journal of Energy Resources Technology, Vol.132, Issue 2, pp. 0210011-0210019.

[22] Christmann, J.B.P. Krätz, L.J. Bart, H.-J. "Falling Film Evaporation with Polymeric Heat Transfer Surfaces," Desalination, Vol. 308, pp. 56-62, January 2013.

[23] Laaber, D. and Bart, H.-J. "Chemical and Pressure Stress Resistance of Polymer Films," Polymer Testing, Vol. 40, pp. 280-285, December 2014.

[24] Yan, X. Li, B. Liu, B. Zhao, J. Wang, Y. Li, H. "Analysis of Improved Novel Hollow Fiber Heat Exchanger", Applied Thermal Engineering, Vol. 67, Issues 1-2, pp. 114-121, June 2011.

[25] API Standard 660, "Shell-and-Tube Heat Exchangers," American Petroleum Institute, Available: http://www.api.org/publications-standards-andstatistics/standards/whatsnew/publication-updates/newrefining-publications/api_std_660 [Accessed: September $15,2015]$.
[26] Rodgers, P. Eveloy, V. and El Sayed, L. "Candidate Thermally Enhanced Polymer Composite Materials for Cooling of Electronic Systems," In Proceedings of the Twentieth International Workshop on Thermal Investigations of ICs and Systems (THERMINIC), Greenwich, London, UK, 2014.

[27] ANSYS, ANSYS DesignModeler, Available http://www.ansys.com/Products/Workflow+Technology/ ANSYS+Workbench+Platform/ANSYS+DesignModeler [Accessed: September 15, 2015].

[28] ANSYS, ANSYS Meshing, Available http://www.ansys.com/Products/Workflow+Technology/ ANSYS+Workbench+Platform/ANSYS+Meshing [Accessed: September 15, 2015].

[29] ANSYS, ANSYS Fluent, Version 16.0, Available: http://www.ansys.com/Products/Simulation+Technology /Fluid+Dynamics/Fluid+Dynamics+Products/ANSYS+F luent [Accessed: September 15, 2015].

[30] ANSYS, ANSYS Mechanical, Version 16.0, Available: http://www.ansys.com/Products/Simulation+Technology /Structural+Analysis/ANSYS+Mechanical [Accessed: September 15, 2015]. 